\title{
A class of nonlocal indefinite differential systems
}

Ping $\mathrm{Li}^{1}$, Meiqiang Feng ${ }^{1 *}$ and Peige Qin ${ }^{1}$

${ }^{\text {} C}$ Correspondence:
meiqiangfeng@sina.com
1 School of Applied Science, Beijing
Information Science \& Technology
University, Beijing, People's Republic
of China
of China

\begin{abstract}
We consider the solvable intervals of two positive parameters $\lambda$ and $\mu$ in which the second-order nonlocal differential system

$$
\left\{\begin{array}{l}
-u^{\prime \prime}=a(t) \varphi u+\lambda \omega(t) f(u), \quad 0<t<1 \\
-\varphi^{\prime \prime}=\mu b(t) u, \quad 0<t<1 \\
u(0)=\int_{0}^{1} g(s) u(s) d s, \quad u^{\prime}(1)=0 \\
\varphi(0)=\varphi(1)=\int_{0}^{1} h(s) \varphi(s) d s
\end{array}\right.
$$

admits multiple positive solutions. The main interest is that the weight functions $a(t)$, $\omega(t)$ and $b(t)$ change sign on $[0,1], \lambda \not \equiv 1, \mu \neq \equiv 1, g \not \equiv 0$ and $h \not \equiv 0$. Our analysis mainly relies on the fixed point technology in cones.
\end{abstract}

Keywords: Positive solutions; Nonlocal indefinite differential systems; Multi-parameter; Fixed point technology

\section{Introduction}

Boundary value problem with integral boundary conditions is a typical nonlocal problem, which arises naturally in hydrodynamic problems [1], semiconductor problems [2], thermal conduction problems [3]. Such problems have been considered by many authors [4-18]. Specifically, Boucherif [19] exploited the fixed point theorem in cones to study the following problem:

$$
\left\{\begin{array}{l}
u^{\prime \prime}(t)=f(t, u(t)), \quad 0<t<1, \\
u(0)-c u^{\prime}(0)=\int_{0}^{1} g_{0}(t) u(t) d t, \\
u(1)-d u^{\prime}(1)=\int_{0}^{1} g_{1}(t) u(t) d t .
\end{array}\right.
$$

The author got several excellent results on the existence of positive solutions to problem (1.1).

In [20], Feng, Ji and Ge began to study the boundary value problem with integral boundary conditions in abstract spaces

$$
\left\{\begin{array}{l}
u^{\prime \prime}(t)+f(t, u(t))=\theta, \quad 0<t<1, \\
u(0)=\int_{0}^{1} g(t) u(t) d t, \quad u(1)=\theta .
\end{array}\right.
$$

(c) The Author(s) 2018. This article is distributed under the terms of the Creative Commons Attribution 4.0 International License (http://creativecommons.org/licenses/by/4.0/), which permits unrestricted use, distribution, and reproduction in any medium, provided you give appropriate credit to the original author(s) and the source, provide a link to the Creative Commons license, and indicate if changes were made. 
Applying the fixed point theory in a cone for strict set contraction operators, the authors investigated the existence, nonexistence and multiplicity of positive solutions for problem (1.2).

At the same time, we see that increasing attention has been paid to the study of nonlocal boundary value problems with parameters (see [21-30]). Especially, let us review several excellent results related to boundary value problems with parameters. In [31], Kong considered the existence and uniqueness of positive solutions for second-order singular boundary value problem

$$
\left\{\begin{array}{l}
u^{\prime \prime}(t)+\lambda f(u(t))=0, \quad t \in(0,1), \\
u(0)=\int_{0}^{1} u(s) d A(s), \quad u(1)=\int_{0}^{1} u(s) d B(s) .
\end{array}\right.
$$

The author examined the uniqueness of the solution and its dependence on the parameter $\lambda$ for problem (1.3) by using the mixed monotone operator theory.

Very recently, Zhang and Feng [32] considered the following second-order differential equations with one-dimensional $p$-Laplacian:

$$
\left\{\begin{array}{l}
\lambda\left(\varphi_{p}\left(u^{\prime}\right)\right)^{\prime}+\omega(t) f(t, u)=0, \quad t \in(0,1), \\
a u(0)-b u^{\prime}(0)=\int_{0}^{1} g(t) u(t) d t, \quad u^{\prime}(1)=0,
\end{array}\right.
$$

where $\lambda>0$ is a parameter, $\varphi_{p}(s)=|s|^{p-2} s, p>1,\left(\varphi_{p}\right)^{-1}=\varphi_{q}, \frac{1}{p}+\frac{1}{q}=1, a, b>0$, $\omega$ is a nonnegative measurable function on $(0,1), \omega \neq 0$ on any open subinterval in $(0,1)$ which may be singular at $t=0$ and/or $t=1$. Using fixed point techniques, the authors obtain some new and more general existence, nonexistence and multiplicity results. In addition, they also studied the dependence of positive solution $u_{\lambda}$ on the parameter $\lambda$.

For the latest development direction of the nonlocal problems, see Refs. [33-37]. However, to the best of our knowledge, the corresponding results for second-order differential system with integral boundary conditions and multiple parameters are not investigated until now.

Moreover, a class of indefinite problems have attracted the attention of Ma and Han [38], López-Gómez and Tellini [39], Boscaggin and Zanolin [40, 41], Sovrano and Zanolin [42], Bravo and Torres [43], Wang and An [44], and Yao [45]. In [38], Ma and Han considered the following boundary value problem:

$$
\left\{\begin{array}{l}
u^{\prime \prime}+\lambda a(t) f(u)=0, \quad 0<t<1, \\
u(0)=u(1)=0
\end{array}\right.
$$

where $a \in C[0,1]$ may change sign, $\lambda$ is a parameter. They proved the existence, multiplicity and stability of positive solutions for problem (1.5) by applying bifurcation techniques.

In [42], applying the shooting method, Sovrano and Zanolin presented a multiplicity result of positive solutions for the Neumann problem

$$
\left\{\begin{array}{l}
u^{\prime \prime}+a(t) f(u)=0, \quad 0<t<1 \\
u(t)>0, \quad t \in[0, T] \\
u^{\prime}(0)=u^{\prime}(T)=0
\end{array}\right.
$$

where the weight function $a \in C[0,1]$ has indefinite sign . 
Recently, Wang and An in [44] dealt with the existence and multiplicity of positive solutions for the second-order differential system

$$
\left\{\begin{array}{l}
-u^{\prime \prime}=a(t) \varphi u+h(t) f(u), \quad 0<t<1 \\
-\varphi^{\prime \prime}=b(t) u, \quad 0<t<1 \\
u(0)=u(1)=0 \\
\varphi(0)=\varphi(1)=0
\end{array}\right.
$$

where $a(t), b(t), g(t)$ are allowed to change sign on $[0,1]$.

Very recently, López-Gómez, Omari, Rivetti $[46,47]$ studied a class of quasilinear indefinite problem

$$
\left\{\begin{array}{l}
-\left(\frac{u^{\prime}}{\sqrt{1+u^{\prime 2}}}\right)^{\prime}=\lambda a(t) f(u), \quad 0<t<1, \\
u^{\prime}(0)=u^{\prime}(1)=0
\end{array}\right.
$$

where $\lambda \in \mathrm{R}$ is a parameter, $a \in L^{1}[0,1]$ changes sign. They derived many results on the existence and the multiplicity of positive (regular) solutions by applying topological degree and variational approach, respectively. For other results on indefinite problems, we refer the reader to Refs. [48-51] and the references cited therein.

To the best of our knowledge, in the literature there are no articles on multiple positive solutions for the analogous of second-order nonlocal differential system with indefinite weights and multiple parameters. More precisely, the study of $\lambda \not \equiv 1, \mu \neq \equiv 1$, and $a(t), b(t)$ and $\omega(t)$ changing sign on $[0,1]$ is still open for the second-order nonlocal differential system

$$
\left\{\begin{array}{l}
-u^{\prime \prime}=a(t) \varphi u+\lambda \omega(t) f(u), \quad 0<t<1 \\
-\varphi^{\prime \prime}=\mu b(t) u, \quad 0<t<1, \\
u(0)=\int_{0}^{1} g(s) u(s) d s, \quad u^{\prime}(1)=0 \\
\varphi(0)=\varphi(1)=\int_{0}^{1} h(s) \varphi(s) d s
\end{array}\right.
$$

where $\lambda>0$ and $\mu>0$ are two parameters, $a(t), b(t), \omega(t)$ may change sign on $J=[0,1]$. We assume that $a, \omega, b, f, g$ and $h$ satisfy

$\left(H_{1}\right) a, \omega, b:[0,1] \rightarrow(-\infty,+\infty)$ are continuous, and there exists a constant $\xi \in(0,1)$ such that

$$
\begin{cases}a(t), \omega(t), b(t) \geq 0, & \forall t \in[0, \xi], \\ a(t)), \omega(t), b(t) \leq 0, & \forall t \in[\xi, 1] .\end{cases}
$$

Moreover, $a(t), \omega(t), b(t)$ do not vanish identically on any subinterval of $[0,1]$.

$\left(H_{2}\right) f \in C\left(\mathrm{R}^{+}, \mathrm{R}^{+}\right)$, and $f(s)>0$ for $s>0$, where $\mathrm{R}^{+}=[0,+\infty)$.

$\left(H_{3}\right) g, h \in L^{1}[0,1]$ are nonnegative on $J$, and $\varrho \in[0,1), v \in[0,1)$, where

$$
\varrho=\int_{0}^{1} g(s) d s, \quad v=\int_{0}^{1} h(s) d s
$$


We call a pair of functions $(u, \varphi)$ with $u, \varphi \in C^{2}(0,1) \cap C[0,1]$ being a solution to system (1.9), if $(u, \varphi)$ satisfies (1.9) for $t \in J$. A solution $(u, \varphi)$ is called a positive solution if, for any $t \in J$, we get $u(t), \varphi(t) \geq 0$ and $u(t), \varphi(t) \not \equiv 0$.

Inspired by the work of the above papers, our aim in the present paper is to investigate the multiplicity of positive solutions of system (1.9). We remark that this is probably the first time that the existence of multiple positive solutions of the second-order nonlocal differential system with indefinite weight and multiple parameters has been studied.

The rest of present article is organized as follows. In Sect. 2, we give an equivalent integral equation of system (1.9) and present several lemmas which are needed throughout this article. Section 3 is devoted to the statement and proof the main results. Several related remarks are given in Sect. 4. Finally, we give an example to illustrate the main results in the final section.

\section{An equivalent formulation of system (1.9)}

In this part, we will aim for changing system (1.9) into an equivalent integral equation, and present several lemmas which play an important role in the proof of our main results.

It is clear that system (1.9) is equivalent to the following two boundary value problems:

$$
\left\{\begin{array}{l}
-u^{\prime \prime}=a(t) \phi u+\lambda \omega(t) f(u), \quad 0<t<1 \\
u(0)=\int_{0}^{1} g(s) u(s) d s, \quad u^{\prime}(1)=0
\end{array}\right.
$$

and

$$
\left\{\begin{array}{l}
-\varphi^{\prime \prime}=\mu b(t) u, \quad 0<t<1, \\
\varphi(0)=\varphi(1)=\int_{0}^{1} h(s) \varphi(s) d s .
\end{array}\right.
$$

Lemma 2.1 Assume that $\left(H_{1}\right)-\left(H_{3}\right)$ hold. Then problem (2.1) has a unique solution $u$ and $u$ can be expressed in the form

$$
u(t)=\int_{0}^{1} H(t, s) a(s) u(s) \phi(s) d s+\lambda \int_{0}^{1} H(t, s) \omega(s) f(u(s)) d s
$$

where

$$
\begin{aligned}
& H(t, s)=G(t, s)+\frac{1}{1-\varrho} \int_{0}^{1} G(s, \tau) g(\tau) d \tau \\
& G(t, s)= \begin{cases}t, & 0 \leq t \leq s \leq 1 \\
s, & 0 \leq s \leq t \leq 1 .\end{cases}
\end{aligned}
$$

Proof The proof of Lemma 2.1 is analogous to that of Lemma 2.1 in [52].

It follows from (2.4) and (2.5) that $H(t, s)$ and $G(t, s)$ have the following properties.

Proposition 2.1 Assume that $\left(H_{3}\right)$ holds. Then we have

$$
G(t, s)>0, \quad H(t, s)>0, \quad \forall t, s \in(0,1)
$$




$$
\begin{aligned}
& G(t, s) \geq 0, \quad H(t, s) \geq 0, \quad \forall t, s \in J, \\
& t G(s, s) \leq G(t, s) \leq G(s, s), \quad t H(s, s) \leq H(t, s) \leq H(s, s), \quad \forall t, s \in J, \\
& G(t, s) \geq \xi G(s, s), \quad H(t, s) \geq \xi H(s, s), \quad \forall t \in[\xi, 1], s \in J .
\end{aligned}
$$

Proof By the definition of $G(t, s)$ and $H(t, s)$, it is not difficult to see that (2.6) and (2.7) hold.

Next, turning to (2.8), if $t \leq s$, then $G(t, s)=t \leq s$; if $t \geq s$, then $G(t, s)=s$. So we see that $G(t, s) \leq s=G(s, s), \forall t, s \in J$.

On the other hand, noticing that $t, s \in J$, if $t \leq s$, then $G(t, s)=t \geq t s$; if $t \geq s$, then $G(t, s)=$ $s \geq s t$. Therefore, we obtain $G(t, s) \geq t s=t G(s, s), \forall t, s \in J$.

Similarly, one can prove that $t H(s, s) \leq H(t, s) \leq H(s, s), \forall t, s \in J$. This gives the proof of (2.8).

If $t \in[\xi, 1]$, it is easy to see that by (2.8), $G(t, s) \geq t G(s, s) \geq \xi G(s, s), H(t, s) \geq t H(s, s) \geq$ $\xi H(s, s), \forall s \in J$. This finishes the proof of (2.9).

Lemma 2.2 Assume that $\left(H_{1}\right)$ and $\left(H_{3}\right)$ hold. Then the problem

$$
\left\{\begin{array}{l}
-\varphi^{\prime \prime}=\mu b(t) u, \quad 0<t<1, \\
\varphi(0)=\varphi(1)=\int_{0}^{1} h(s) \varphi(s) d s
\end{array}\right.
$$

has a unique solution $\varphi$ given by

$$
\varphi(t)=\mu \int_{0}^{1} H^{*}(t, s) b(s) u(s) d s
$$

where

$$
\begin{aligned}
H^{*}(t, s) & =G^{*}(t, s)+\frac{1}{1-v} \int_{0}^{1} G^{*}(s, \tau) h(\tau) d \tau, \\
G^{*}(t, s) & = \begin{cases}t(1-s), & 0 \leq t \leq s \leq 1 \\
s(1-t), & 0 \leq s \leq t \leq 1\end{cases}
\end{aligned}
$$

Proof The proof of Lemma 2.2 is similar to that of Lemma 2.1 in [52].

Similar to the proof of Proposition 2.1, we can get Proposition 2.2.

Proposition 2.2 Assume that $\left(H_{3}\right)$ holds. Then we have

$$
\begin{aligned}
& G^{*}(t, s) \geq 0, \quad H^{*}(t, s) \geq 0, \quad \forall t, s \in J, \\
& G^{*}(t, s)>0, \quad H^{*}(t, s)>0, \quad \forall t, s \in(0,1), \\
& G^{*}(t, t) G^{*}(s, s) \leq G^{*}(t, s) \leq G^{*}(s, s)=s(1-s) \leq \frac{1}{4}, \quad \forall t, s \in J, \\
& G^{*}(t, t) H^{*}(s, s) \leq H^{*}(t, s) \leq H^{*}(s, s), \quad \forall t, s \in J, \\
& G^{*}(t, s) \geq \xi^{2} G^{*}(s, s), \quad H^{*}(t, s) \geq \xi^{2} H(s, s), \quad \forall t \in[\xi, 1-\xi], s \in J,
\end{aligned}
$$




$$
\rho G^{*}(s, s) \leq H^{*}(t, s) \leq \gamma G^{*}(s, s)=\gamma e(s) \leq \frac{1}{4} \gamma, \quad \forall t, s \in J,
$$

where

$$
\rho=\frac{\int_{0}^{1} G^{*}(\tau, \tau) g(\tau) d \tau}{1-v}, \quad \gamma=\frac{1}{1-v} .
$$

Remark 2.1 Letting $(u, \varphi)$ be a solution of system (1.9), then from Lemma 2.1 and Lemma 2.2, we have

$$
\begin{aligned}
u(t)= & \mu \int_{0}^{1} \int_{0}^{1} H(t, s) H^{*}(s, \tau) a(s) b(\tau) u(s) u(\tau) d \tau d s \\
& +\lambda \int_{0}^{1} H(t, s) \omega(s) f(u(s)) d s
\end{aligned}
$$

and $\varphi$ is defined in (2.11).

For the weight functions $a(t), b(t), \omega(t)$, we define $a^{ \pm}(t), b^{ \pm}(t), \omega^{ \pm}(t): J \rightarrow[0,+\infty)$ as

$$
\begin{array}{ll}
a^{+}(t)=\max \{a(t), 0\}, & a^{-}(t)=-\min \{a(t), 0\}, \\
b^{+}(t)=\max \{b(t), 0\}, & b^{-}(t)=-\min \{b(t), 0\}, \\
\omega^{+}(t)=\max \{\omega(t), 0\}, & \omega^{-}(t)=-\min \{\omega(t), 0\},
\end{array}
$$

consequently

$$
a(t)=a^{+}(t)-a^{-}(t), \quad b(t)=b^{+}(t)-b^{-}(t), \quad \omega(t)=\omega^{+}(t)-\omega^{-}(t), \quad \forall t \in J .
$$

Moreover, to obtain the existence of positive solution of system (1.9), we make the following hypotheses:

$\left(H_{4}\right)$ There exists $0<\sigma_{1}<\xi$ such that

$$
\sigma_{1} \int_{\sigma_{1}}^{\xi} G(t, s) b^{+}(s) d s \geq \xi \int_{\xi}^{1} G(t, s) b^{-}(s) d s
$$

$\left(H_{5}\right)$ There exists $0<\sigma_{2}<\xi$ such that

$$
\sigma_{2} \int_{\sigma_{2}}^{\xi} H(t, s) H^{*}(s, s) a^{+}(s) d s \geq \frac{\xi}{1-v} \int_{\xi}^{1} H(t, s) a^{-}(s) d s .
$$

$\left(H_{6}\right)$ There exists $0<l \leq 1$ such that

$$
f(u) \geq l \phi(u), \quad u \in[0,+\infty),
$$

where $\phi(u)=\max \{f(\beta): 0 \leq \beta \leq u\}$.

$\left(H_{7}\right)$ There exist $0<\theta<+\infty, \theta \neq 1$ and $k_{1}, k_{2}>0$ such that

$$
k_{1} u^{\theta} \leq f(u) \leq k_{2} u^{\theta}, \quad u \in[0,+\infty) .
$$


$\left(H_{8}\right)$ There exists $0<\sigma_{3}<\xi$ such that

$$
l^{2} k_{1} \sigma_{3}^{\theta} \int_{\sigma_{3}}^{\xi} H(t, s) \omega^{+}(s) d s \geq k_{2} \xi^{\theta} \int_{\xi}^{1} H(t, s) \omega^{-}(s) d s
$$

We denote

$$
\begin{aligned}
& C_{0}^{+}[0,1]=\left\{u \in C[0,1]: \min _{t \in J} u(t) \geq 0, u(0)=\int_{0}^{1} g(s) u(s) d s, u^{\prime}(1)=0\right\} \\
& K=\left\{u \in C_{0}^{+}[0,1]: \text { and } \mathrm{u} \text { is concave on }[0, \xi] \text { and convex on }[\xi, 1]\right\} .
\end{aligned}
$$

If $u \in K$, it is not difficult to see that $\|u\|=\max _{0 \leq t \leq \xi} u(t)$.

Also, for a positive number $r$, we define $\Omega_{r}$ by

$$
\Omega_{r}=\{u \in K:\|u\|<r\}
$$

and then we get $\partial \Omega_{r}=\{u \in K:\|u\|=r\}$.

Define a map $T: K \rightarrow C[0,1]$ by

$$
\begin{aligned}
(T u)(t)= & \mu \int_{0}^{1} \int_{0}^{1} H(t, s) H^{*}(s, \tau) a(s) u(s) b(\tau) u(\tau) d \tau d s \\
& +\lambda \int_{0}^{1} H(t, s) \omega(s) f(u(s)) d s .
\end{aligned}
$$

It follows from Remark 2.1 and (2.18) that Lemma 2.3 holds.

Lemma 2.3 Assume that $\left(H_{1}\right)-\left(H_{8}\right)$ hold. Then $(u, \varphi)$ is a positive solution of system (1.9) if and only if $u$ is a fixed point of operator $T$ in $K$, and $\varphi$ satisfies (2.11).

Lemma 2.4 Assume that $\left(H_{1}\right)-\left(H_{8}\right)$ hold. Then $T(K) \subset K$, and $T: K \rightarrow K$ is completely continuous.

Proof For any $u \in K$, we firstly prove that

$$
\int_{0}^{1} G(t, s) b(s) u(s) d s \geq \int_{0}^{\sigma_{0}} G(t, s) b^{+}(s) u(s) d s, \quad t \in J
$$

Define a function $q:[0,1] \rightarrow[0,1]$ as follows:

$$
\begin{aligned}
& \text { if } u(1)=0, \quad \text { then } q(t)=\min \left\{\frac{t}{\xi}, \frac{1-t}{1-\xi}\right\}, \quad \forall t \in J ; \\
& \text { if } u(1)>0, \quad \text { then } q(t)=\min \left\{\frac{t}{\xi}, 1\right\}, \quad \forall t \in J .
\end{aligned}
$$

So, we get $\min _{0<\sigma \leq t \leq \xi} q(t)=\frac{\sigma}{\xi}, \max _{\xi \leq t \leq 1} q(t)=1$.

Since $u \in K$, then $u$ is concave on $[0, \xi]$ and convex on $[\xi, 1]$. Noticing that $u(0)=$ $\int_{0}^{1} g(t) u(t) d t$ and $u^{\prime}(1)=0$, we get

$$
u(t) \geq q(t) u(\xi), \quad t \in[0, \xi], \quad u(t) \leq q(t) u(\xi), \quad t \in[\xi, 1]
$$


Hence, for $t \in J$, by assumption $\left(H_{4}\right)$, we have

$$
\begin{aligned}
& \int_{0}^{1} G(t, s) b(s) u(s) d s-\int_{0}^{\sigma_{1}} G(t, s) b^{+}(s) u(s) d s \\
& \quad=\int_{\sigma_{1}}^{\xi} G(t, s) b^{+}(s) u(s) d s-\int_{\xi}^{1} G(t, s) b^{-}(s) u(s) d s \\
& \quad \geq \int_{\sigma_{1}}^{\xi} G(t, s) b^{+}(s) q(s) u(\xi) d s-\int_{\xi}^{1} G(t, s) b^{-}(s) q(s) u(\xi) d s \\
& \quad \geq u(\xi)\left[\min _{s \in\left[\sigma_{1}, \xi\right]} q(s) \int_{\sigma_{1}}^{\xi} G(t, s) b^{+}(s) d s-\max _{s \in[\xi, 1]} q(s) \int_{\xi}^{1} G(t, s) b^{-}(s) d s\right] \\
& \quad=u(\xi)\left[\frac{\sigma_{1}}{\xi} \int_{\sigma_{1}}^{\xi} G(t, s) b^{+}(s) d s-\int_{\xi}^{1} G(t, s) b^{-}(s) d s\right] \\
& \quad \geq 0
\end{aligned}
$$

which shows that (2.23) holds.

Next, for any $u \in K$, we prove

$$
\begin{aligned}
& \int_{0}^{1} \int_{0}^{1} H(t, s) H^{*}(s, \tau) a(s) u(s) b(\tau) u(\tau) d \tau d s \\
& \quad \geq \int_{0}^{\sigma_{2}} \int_{0}^{1} H(t, s) H^{*}(s, \tau) a^{+}(s) u(s) b(\tau) u(\tau) d \tau d s, \quad t \in J
\end{aligned}
$$

Since $\int_{0}^{1} G(t, s) b(s) u(s) d s \geq 0, t \in J$, it follows from (2.14) and $\left(H_{5}\right)$ that

$$
\begin{aligned}
& \int_{0}^{1} \int_{0}^{1} H(t, s) H^{*}(s, \tau) a(s) u(s) b(\tau) u(\tau) d \tau d s \\
& \quad-\int_{0}^{\sigma_{2}} \int_{0}^{1} H(t, s) H^{*}(s, \tau) a^{+}(s) u(s) b(\tau) u(\tau) d \tau d s \\
&=\int_{\sigma_{2}}^{\xi} \int_{0}^{1} H(t, s) H^{*}(s, \tau) a^{+}(s) u(s) b(\tau) u(\tau) d \tau d s \\
& \quad-\int_{\xi}^{1} \int_{0}^{1} H(t, s) H^{*}(s, \tau) a^{-}(s) u(s) b(\tau) u(\tau) d \tau d s \\
& \geq \int_{\sigma_{2}}^{\xi} H(t, s) a^{+}(s) q(s) u(\xi) \int_{0}^{1} H^{*}(s, \tau) b(\tau) u(\tau) d \tau d s \\
& \quad-\int_{\xi}^{1} H(t, s) a^{-}(s) q(s) u(\xi) \int_{0}^{1} H^{*}(s, \tau) b(\tau) u(\tau) d \tau d s \\
& \geq \min _{s \in\left[\sigma_{2}, \xi\right]} q(s) u(\xi) \int_{\sigma_{2}}^{\xi} H(t, s) a^{+}(s) d s \int_{0}^{1} H^{*}(s, \tau) b(\tau) u(\tau) d \tau \\
& \quad-\max _{s \in[\xi, 1]} q(s) u(\xi) \int_{\xi}^{1} H(t, s) a^{-}(s) d s \int_{0}^{1} H^{*}(s, \tau) b(\tau) u(\tau) d \tau \\
& \geq u(\xi) \frac{\sigma_{2}}{\xi} \int_{\sigma_{2}}^{\xi} H(t, s) a^{+}(s) \int_{0}^{1} H^{*}(s, \tau) b(\tau) u(\tau) d \tau d s
\end{aligned}
$$




$$
\begin{aligned}
& -u(\xi) \int_{\xi}^{1} H(t, s) a^{-}(s) \int_{0}^{1} H^{*}(s, \tau) b(\tau) u(\tau) d \tau d s \\
\geq & u(\xi) \frac{\sigma_{2}}{\xi} \int_{\sigma_{2}}^{\xi} H(t, s) a^{+}(s) \int_{0}^{1} H^{*}(s, s) G(\tau, \tau) b(\tau) u(\tau) d \tau d s \\
& -u(\xi) \int_{\xi}^{1} H(t, s) a^{-}(s) \int_{0}^{1} \frac{1}{1-v} G(\tau, \tau) b(\tau) u(\tau) d \tau d s \\
= & u(\xi) \int_{0}^{1} G(\tau, \tau) b(\tau) u(\tau) d \tau\left[\frac{\sigma_{2}}{\xi} \int_{\sigma_{2}}^{\xi} H(t, s) H^{*}(s, s) a^{+}(s) d s\right. \\
& \left.-\frac{1}{1-v} \int_{\xi}^{1} H(t, s) a^{-}(s) d s\right] .
\end{aligned}
$$

This finishes the proof of (2.24).

Thirdly, for any $u \in K$, we show

$$
\int_{0}^{1} H(t, s) \omega(s) f(u(s)) d s \geq \int_{0}^{\sigma_{3}} H(t, s) \omega^{+}(s) f(u(s)) d s, \quad t \in J
$$

In fact, $t \in J, u \in K$, it is easy to see by $\left(H_{6}\right)-\left(H_{8}\right)$ that

$$
\begin{array}{rl}
\int_{0}^{1} & H(t, s) g(s) f(x(s)) d s-\int_{0}^{\sigma_{3}} H(t, s) g^{+}(s) f(x(s)) d s \\
& =\int_{\sigma_{3}}^{\xi} H(t, s) g^{+}(s) f(x(s)) d s-\int_{\xi}^{1} H(t, s) g^{-}(s) f(x(s)) d s \\
& \geq l \int_{\sigma_{3}}^{\xi} H(t, s) g^{+}(s) \varphi(u(s)) d s-\int_{\xi}^{1} H(t, s) g^{-}(s) \varphi(u(s)) d s \\
\geq & l \int_{\sigma_{3}}^{\xi} H(t, s) g^{+}(s) \varphi(e(s) u(\xi)) d s-\int_{\xi}^{1} H(t, s) g^{-}(s) \varphi(e(s) u(\xi)) d s \\
\geq & l \int_{\sigma_{3}}^{\xi} H(t, s) g^{+}(s) f(e(s) u(\xi)) d s-\frac{1}{l} \int_{\xi}^{1} H(t, s) g^{-}(s) f(e(s) u(\xi)) d s \\
\geq & l \int_{\sigma_{3}}^{\xi} H(t, s) g^{+}(s) k_{1} e^{\alpha}(s) u^{\theta}(\xi) d s-\frac{1}{l} \int_{\xi}^{1} H(t, s) g^{-}(s) k_{2} e^{\theta}(s) u^{\theta}(\xi) d s \\
\geq & l\left[\min _{s \in\left[\sigma_{3}, \xi\right]} e(s)\right]^{\theta} \int_{\sigma_{3}}^{\xi} H(t, s) g^{+}(s) k_{1} u^{\theta}(\xi) d s \\
& -\frac{1}{l}\left[\min _{s \in[\xi, 1]} e(s)\right]^{\theta} \int_{\xi}^{1} H(t, s) g^{-}(s) k_{2} u^{\theta}(\xi) d s \\
\geq & u^{\theta}(\xi)\left[l k_{1} \frac{\sigma_{3}^{\theta}}{\xi^{\theta}} \int_{\sigma_{3}}^{\xi} H(t, s) g^{+}(s) d s-\frac{1}{l} k_{2} \int_{\xi}^{1} H(t, s) g^{-}(s) d s\right] \\
\geq & 0,
\end{array}
$$

which shows that (2.25) holds. 
Thus, for $u \in K$, we have

$$
\begin{aligned}
(T u)(t)= & \mu \int_{0}^{1} \int_{0}^{1} H(t, s) H^{*}(s, \tau) a(s) u(s) b(\tau) u(\tau) d \tau d s+\lambda \int_{0}^{1} H(t, s) \omega(s) f(u(s)) d s \\
\geq & \mu \int_{0}^{\sigma_{2}} H(t, s) a^{+}(s) u(s) \int_{0}^{1} H^{*}(s, \tau) b(\tau) u(\tau) d \tau d s \\
& +\lambda \int_{0}^{\sigma_{3}} H(t, s) \omega^{+}(s) f(u(s)) d s \\
\geq & 0 .
\end{aligned}
$$

Moreover, by direct calculating, we get

$$
\begin{aligned}
& (T u)(0)=\int_{0}^{1} g(t)(T u)(t) d t, \quad(T u)^{\prime}(1)=0, \\
& (T u)^{\prime \prime}(t)=-\mu a^{+}(t) u(t) \int_{0}^{1} H^{*}(t, s) b(s) u(s) d s-\lambda \omega^{+}(t) f(u(t)) \leq 0, \quad t \in[0, \xi], \\
& (T u)^{\prime \prime}(t)=\mu a^{-}(t) u(t) \int_{0}^{1} H^{*}(t, s) b(s) u(s) d s+\lambda \omega^{-}(t) f(u(t)) \geq 0, \quad t \in[\xi, 1],
\end{aligned}
$$

which shows that $T x$ is concave on $[0, \xi]$ and convex on $[\xi, 1]$. It follows that $T: K \rightarrow K$.

Finally, by standard methods and Arzelà-Ascoli theorem one can prove that operator $T$ is completely continuous. So it is omitted.

Remark 2.2 In [44] and [45], it is not difficult to see that the function $q$ plays an important role in the proof of completely continuous operator. If $u(0)=u(1)=0$, then we can define $q(t)=\min \left\{\frac{t}{\xi}, \frac{1-t}{1-\xi}\right\}$. However, if $u(0)=u(1)=A \neq 0$, then the above definition of $q$ is invalid. This shows that when $u(0)=\int_{0}^{1} g(s) u(s) d s, u^{\prime}(1)=0$, we require a special technique to give a fine definition of $q$.

In fact, a fine definition of $q$ is very difficult to give when $u(0)=\int_{0}^{1} g(s) u(s) d s, u^{\prime}(1)=0$. This is probably the main reason that there is almost no paper studying the existence of positive solutions for the class of second-order nonlocal differential systems with indefinite weights and multiple parameters.

Remark 2.3 When we consider nonlocal differential systems with indefinite weights, another difficulty is to prove $T: K \rightarrow K$; for detail to see the proof of Lemma 2.4.

Lemma 2.5 (Theorem 2.3.4 of [53], Fixed point theorem of cone expansion and compression of norm type) Let $\Omega_{1}$ and $\Omega_{2}$ be two bounded open sets in a real Banach space $E$ such that $0 \in \Omega_{1}$ and $\bar{\Omega}_{1} \subset \Omega_{2}$. Let operator $T: P \cap\left(\bar{\Omega}_{2} \backslash \Omega_{1}\right) \rightarrow P$ be completely continuous, where $P$ is a cone in E. Suppose that one of the two conditions

(i) $\|T x\| \leq\|x\|, \forall x \in P \cap \partial \Omega_{1}$ and $\|T x\| \geq\|x\|, \forall x \in P \cap \partial \Omega_{2}$, and

(ii) $\|T x\| \geq\|x\|, \forall x \in P \cap \partial \Omega_{1}$, and $\|T x\| \leq\|x\|, \forall x \in P \cap \partial \Omega_{2}$, is satisfied. Then $T$ has at least one fixed point in $P \cap\left(\bar{\Omega}_{2} \backslash \Omega_{1}\right)$.

\section{Main results}

In this part, applying Lemma 2.5, we get the solvable intervals of positive parameters $\lambda$ and $\mu$ in which system (1.9) admits multiple positive solutions. 
Theorem 3.1 Assume that $\left(H_{1}\right)-\left(H_{8}\right)$ hold. Then we get:

(I) If $\theta>1$, then there exist $\lambda^{*}, \mu_{*}, \mu^{*}>0$ such that, for $\lambda \in\left[\lambda^{*},+\infty\right)$ and $\mu \in\left[\mu_{*}, \mu^{*}\right]$, system (1.9) admits at least two positive solutions.

(II) If $0<\theta<1$, then there exist $\lambda^{* *}, \mu^{* *}>0$ such that, for $\lambda \in\left(0, \lambda^{* *}\right]$ and $\mu \in\left(0, \mu^{* *}\right]$, system (1.9) admits at least two positive solutions.

Proof Part (I). Considering the case $\theta>1$, it follows from $\left(H_{7}\right)$ that

$$
\lim _{u \rightarrow 0} \frac{f(u)}{u} \leq \lim _{u \rightarrow 0} \frac{k_{2} u^{\theta}}{u}=0,
$$

which shows that there exists a $r^{\prime}>0$ such that

$$
f(u) \leq \varepsilon_{1} u, \quad 0 \leq u \leq r^{\prime},
$$

where $\varepsilon_{1}$ satisfies

$$
2 \lambda \varepsilon_{1} \int_{0}^{\xi} H(s, s) g^{+}(s) d s<1 .
$$

Let

$$
\begin{aligned}
& A=\mu \int_{0}^{\xi} \int_{0}^{\xi} H(s, s) H^{*}(s, \tau) a^{+}(s) b^{+}(\tau) d \tau d s, \\
& A^{*}=\int_{0}^{\xi} \int_{0}^{\xi} H(s, s) H^{*}(s, \tau) a^{+}(s) b^{+}(\tau) d \tau d s,
\end{aligned}
$$

and choose $r=\min \left\{(2 A)^{-1}, \frac{r^{\prime}}{2}\right\}$.

Then, for any $u \in K \cap \partial \Omega_{r}$, noticing (2.8), we get

$$
\begin{aligned}
(T u)(t)= & \mu \int_{0}^{1} \int_{0}^{1} H(t, s) H^{*}(s, \tau) a(s) b(\tau) u(s) u(\tau) d \tau d s \\
& +\lambda \int_{0}^{1} H(t, s) g(s) f(u(s)) d s \\
= & \mu \int_{0}^{\xi} \int_{0}^{1} H(t, s) H^{*}(s, \tau) a^{+}(s) b(\tau) u(s) u(\tau) d \tau d s \\
& -\mu \int_{\xi}^{1} \int_{0}^{1} H(t, s) H^{*}(s, \tau) a^{-}(s) b(\tau) u(s) u(\tau) d \tau d s \\
& +\lambda \int_{0}^{\xi} H(t, s) g^{+}(s) f(u(s)) d s-\lambda \int_{\xi}^{1} H(t, s) g^{-}(s) f(u(s)) d s \\
\leq & \mu \int_{0}^{\xi} \int_{0}^{1} H(t, s) H^{*}(s, \tau) a^{+}(s) b(\tau) u(s) u(\tau) d \tau d s \\
& +\lambda \int_{0}^{\xi} H(t, s) g^{+}(s) f(u(s)) d s \\
= & \mu \int_{0}^{\xi} \int_{0}^{\xi} H(t, s) H^{*}(s, \tau) a^{+}(s) b^{+}(\tau) u(s) u(\tau) d \tau d s
\end{aligned}
$$




$$
\begin{aligned}
& -\mu \int_{0}^{\xi} \int_{0}^{\xi} H(t, s) H^{*}(s, \tau) a^{+}(s) b^{-}(\tau) u(s) u(\tau) d \tau d s \\
& +\lambda \int_{0}^{\xi} H(t, s) g^{+}(s) f(u(s)) d s \\
\leq & \mu \int_{0}^{\xi} \int_{0}^{\xi} H(s, s) H^{*}(s, \tau) a^{+}(s) b^{+}(\tau) u(s) u(\tau) d \tau d s \\
& +\lambda \int_{0}^{\xi} H(s, s) g^{+}(s) f(u(s)) d s \\
\leq & \mu \int_{0}^{\xi} \int_{0}^{\xi} H(s, s) H^{*}(s, \tau) a^{+}(s) b^{+}(\tau) u(s) u(\tau) d \tau d s \\
& +\lambda \int_{0}^{\xi} H(s, s) g^{+}(s) \varepsilon_{1} u(s) d s \\
\leq & \mu \int_{0}^{\xi} \int_{0}^{\xi} H(s, s) H^{*}(s, \tau) a^{+}(s) b^{+}(\tau) d \tau d s\|u\|^{2} \\
& +\lambda \varepsilon_{1} \int_{0}^{\xi} H(s, s) g^{+}(s) d s\|u\| \\
< & \frac{1}{2}\|x\|+\frac{1}{2}\|u\| \\
= & \|u\|,
\end{aligned}
$$

which shows that

$$
\|T u\|<\|u\|, \quad \forall u \in K \cap \partial \Omega_{r} .
$$

On the other hand, noticing that $\theta>1$, it follows from $\left(H_{7}\right)$ that

$$
\lim _{u \rightarrow \infty} \frac{f(u)}{u} \geq \lim _{u \rightarrow \infty} \frac{k_{1} u^{\alpha}}{u}=\infty
$$

which shows that there exists a $R^{\prime}>0$ such that

$$
f(u) \geq \varepsilon_{2} u, \quad u \geq R^{\prime}
$$

where $\varepsilon_{2}$ satisfies

$$
\sigma_{2} \lambda \varepsilon_{2} \Gamma \int_{\frac{\sigma_{3}}{2}}^{\sigma_{3}} H(s, s) g^{+}(s) d s>1
$$

and

$$
\Gamma=\min _{\frac{\sigma_{3}}{2} \leq t \leq \sigma_{3}} \delta(t)>0, \quad \delta(t)=\min \left\{\frac{t}{\xi}, \frac{\xi-t}{\xi}\right\}, \quad t \in[0, \xi]
$$

If $u \in K$, then from the concavity on $[0, \xi]$, it follows that

$$
u(t) \geq \delta(t)\|u\|, \quad t \in[0, \xi]
$$


Let $B$ and $B^{*}$ denote the following constants:

$$
\begin{aligned}
& B=\mu \frac{\sigma_{2}}{2} \min _{\frac{\sigma_{2}}{2} \leq t \leq \sigma_{2}} \delta(t) \min _{\frac{\sigma_{1}}{2} \leq t \leq \sigma_{1}} \delta(t) \int_{\frac{\sigma_{2}}{2}}^{\sigma_{2}} \int_{\frac{\sigma_{1}}{2}}^{\sigma_{1}} H(s, s) H^{*}(s, \tau) a^{+}(s) b^{+}(\tau) d \tau d s>0, \\
& B^{*}=\frac{\sigma_{2}}{2} \min _{\frac{\sigma_{2}}{2} \leq t \leq \sigma_{2}} \delta(t) \min _{\frac{\sigma_{1}}{2} \leq t \leq \sigma_{1}} \delta(t) \int_{\frac{\sigma_{2}}{2}}^{\sigma_{2}} \int_{\frac{\sigma_{1}}{2}}^{\sigma_{1}} H(s, s) H^{*}(s, \tau) a^{+}(s) b^{+}(\tau) d \tau d s>0 .
\end{aligned}
$$

Noticing that, for all $d \in(0, \xi), \min _{\frac{d}{2} \leq t \leq d} \delta(t)=\min \left\{\frac{d}{2 \xi}, \frac{\xi-d}{\xi}\right\}>0, B$ and $B^{*}$ are defined well.

Setting $R=\max \left\{(2 B)^{-1}, \frac{R^{\prime}}{\Gamma}, r^{\prime}\right\}+1$, it follows that $\mu>\mu^{\prime}=\frac{1}{2 B^{*} R}$, and then, for any $x \in$ $K \cap \partial \Omega_{R}$, it follows from (3.7) that

$$
u(t) \geq \min _{\frac{\sigma_{3}}{2} \leq t \leq \sigma_{3}} \delta(t)\|u\| \geq \Gamma R>R^{\prime},
$$

and then it follows from (2.8), (2.9), (2.20), (2.21), (3.7) and (3.8) that

$$
\begin{aligned}
\|T u\|= & \max _{t \in J}\left\{\mu \int_{0}^{1} \int_{0}^{1} H(t, s) H^{*}(s, \tau) a(s) b(\tau) u(s) u(\tau) d \tau d s\right. \\
& \left.+\lambda \int_{0}^{1} G(t, s) g(s) f(u(s)) d s\right\} \\
\geq & \max _{t \in J}\left\{\mu \int_{0}^{\sigma_{2}} H(t, s) a^{+}(s) u(s) \int_{0}^{1} H^{*}(s, \tau) b(\tau) u(\tau) d \tau d s\right. \\
& \left.+\lambda \int_{0}^{\sigma_{3}} G(t, s) g^{+}(s) f(u(s)) d s\right\} \\
\geq & \mu \min _{\frac{\sigma_{2}}{2} \leq t \leq \sigma_{2}} \int_{\frac{\sigma_{2}}{2}}^{\sigma_{2}} H(t, s) a^{+}(s) u(s) \int_{\frac{\sigma_{1}}{2}}^{\sigma_{1}} H^{*}(s, \tau) b^{+}(\tau) u(\tau) d \tau d s \\
& +\min _{\frac{\sigma_{2}}{2} \leq t \leq \sigma_{2}} \lambda \int_{\frac{\sigma_{3}}{2}}^{\sigma_{3}} H(t, s) g^{+}(s) f(u(s)) d s \\
\geq & \mu \frac{\sigma_{2}}{2} \int_{\frac{\sigma_{2}}{2}}^{\sigma_{2}} H(s, s) a^{+}(s) \delta(s)\|u\| \int_{\frac{\sigma_{1}}{2}}^{\sigma_{1}} H^{*}(s, \tau) b^{+}(\tau) \delta(\tau)\|u\| d \tau d s \\
& +\lambda \frac{\sigma_{2}}{2} \int_{\frac{\sigma_{3}}{2}}^{\sigma_{3}} H(s, s) g^{+}(s) \varepsilon_{2} u(s) d s \\
\geq & \mu \frac{\sigma_{2}}{2} \int_{\frac{\sigma_{2}}{2}}^{\sigma_{2}} H(s, s) a^{+}(s) \delta(s)\|u\| \int_{\frac{\sigma_{1}}{2}}^{\sigma_{1}} H^{*}(s, \tau) b^{+}(\tau) \delta(\tau)\|u\| d \tau d s \\
& +\lambda \frac{\sigma_{2}}{2} \int_{\frac{\sigma_{3}}{2}}^{\sigma_{3}} H(s, s) g^{+}(s) \varepsilon_{2} u(s) d s \\
\geq & B\|u\|^{2}+\lambda \frac{\sigma_{2}}{2} \varepsilon_{2} \min _{\frac{\sigma_{3}}{2} \leq t \leq \sigma_{3}} \delta(t) \int_{\frac{\sigma_{3}}{2}}^{\sigma_{3}} H(s, s) g^{+}(s) d s\|u\| \\
= & \|u\| . \\
& \|u\|+\frac{1}{2}\|u\|
\end{aligned}
$$




\section{Consequently,}

$$
\|T u\|>\|u\|, \quad u \in K \cap \partial \Omega_{R} .
$$

Let

$$
\lambda^{*}=\frac{\left(4 D A^{*}-2 B^{*}\right) \eta}{2 D A^{*} \sigma_{2} \sigma_{\eta} \int_{\frac{\sigma_{3}}{2}}^{\sigma_{3}} H(s, s) g^{+}(s) d s}, \quad \mu^{*}=\frac{1}{2 D A^{*} \eta},
$$

where $0<\eta<r, D=\frac{r^{2}}{\eta^{2}}, A^{*}$ is defined in (3.3), and $B^{*}$ is defined in (3.9).

We define $\sigma_{\eta}$ by

$$
\sigma_{\eta}=\min _{\Gamma \eta \leq u \leq \eta}\{f(u)\}>0
$$

where $\Gamma$ is defined in (3.6). Noticing $\left(H_{2}\right)$, then $\sigma_{\eta}$ is defined well and $\sigma_{\eta}>0$. So, for any $u \in K \cap \partial \Omega_{\eta}$, we get

$$
\Gamma \eta \leq \Gamma\|u\| \leq \min _{\frac{\sigma_{3}}{2} \leq t \leq \sigma_{3}} u(t) \leq u(t) \leq \eta, \quad \frac{\sigma_{3}}{2} \leq t \leq \sigma_{3},
$$

and hence, we get

$$
\begin{aligned}
\|T u\|= & \max _{t \in J}\left\{\mu \int_{0}^{1} \int_{0}^{1} H(t, s) H^{*}(s, \tau) a(s) b(\tau) u(s) u(\tau) d \tau d s\right. \\
& \left.+\lambda \int_{0}^{1} G(t, s) g(s) f(u(s)) d s\right\} \\
\geq & \max _{t \in J}\left\{\mu \int_{0}^{\sigma_{2}} H(t, s) a^{+}(s) u(s) \int_{0}^{1} H^{*}(s, \tau) b(\tau) u(\tau) d \tau d s\right. \\
& \left.+\lambda \int_{0}^{\sigma_{3}} G(t, s) g^{+}(s) f(u(s)) d s\right\} \\
\geq & \mu \min _{\frac{\sigma_{2}}{2} \leq t \leq \sigma_{2}} \int_{\frac{\sigma_{2}}{2}}^{\sigma_{2}} H(t, s) a^{+}(s) u(s) \int_{\frac{\sigma_{1}}{2}}^{\sigma_{1}} H^{*}(s, \tau) b^{+}(\tau) u(\tau) d \tau d s \\
& +\min _{\frac{\sigma_{2}}{2} \leq t \leq \sigma_{2}} \lambda \int_{\frac{\sigma_{3}}{2}}^{\sigma_{3}} H(t, s) g^{+}(s) f(u(s)) d s \\
\geq & \mu \frac{\sigma_{2}}{2} \int_{\frac{\sigma_{2}}{2}}^{\sigma_{2}} H(s, s) a^{+}(s) \delta(s)\|u\| \int_{\frac{\sigma_{1}}{2}}^{\sigma_{1}} H^{*}(s, \tau) b^{+}(\tau) \delta(\tau)\|u\| d \tau d s \\
& +\lambda \frac{\sigma_{2}}{2} \int_{\frac{\sigma_{3}}{2}}^{\sigma_{3}} H(s, s) g^{+}(s) \sigma_{\eta} d s \\
\geq & \mu^{*} B^{*}\|u\|^{2}+\lambda^{*} \frac{\sigma_{2}}{2} \sigma_{\eta} \int_{\frac{\sigma_{3}}{2}}^{\sigma_{3}} H(s, s) g^{+}(s) d s \\
\geq & \frac{B^{*}}{2 D A^{*}}\|u\|+\frac{2 D A^{*}-B^{*}}{2 D A^{*}}\|u\| \\
= & \|u\| .
\end{aligned}
$$


This shows

$$
\|T u\| \geq\|u\|, \quad u \in K \cap \partial \Omega_{\eta} .
$$

Let $\mu_{*}>\max \left\{\mu^{\prime}, \mu^{*}\right\}, \mu^{*}=\frac{1}{2 A^{*} r}$, then, for $\lambda \in\left[\lambda^{*},+\infty\right)$ and $\mu \in\left[\mu_{*}, \mu^{*}\right]$, applying Lemma 2.5 to (3.5), (3.10) and (3.11) shows that $T$ has at least two fixed points in $K \cap\left(\bar{\Omega}_{R} \backslash \bar{\Omega}_{r}\right)$ and $K \cap\left(\Omega_{r} \backslash \Omega_{\eta}\right)$. Thus it follows that system (1.9) admits at least two positive solutions $\left(u_{1}, \varphi_{1}\right)$ and $\left(u_{2}, \varphi_{2}\right)$ with $u_{1} \in K \cap\left(\bar{\Omega}_{R} \backslash \bar{\Omega}_{r}\right), u_{2} \in K \cap\left(\Omega_{r} \backslash \Omega_{\eta}\right)$, and

$$
\varphi_{i}=\mu \int_{0}^{1} H^{*}(t, s) b(s) u_{i}(s) d s, \quad i=1,2 .
$$

Part (II). Next turning to $0<\theta<1$, it follows from $\left(H_{7}\right)$ that

$$
\lim _{u \rightarrow 0} \frac{f(u)}{u} \geq \lim _{u \rightarrow 0} \frac{k_{1} u^{\theta}}{u}=+\infty,
$$

which shows that there exists a positive constant $r>0$ such that

$$
f(u) \geq \varepsilon_{3} u, \quad 0 \leq u \leq r
$$

where $\varepsilon_{3}$ satisfies

$$
\lambda \frac{\sigma_{2}}{2} \varepsilon_{3} \min _{\frac{\sigma_{3}}{2} \leq t \leq \sigma_{3}} \delta(t) \int_{\frac{\sigma_{3}}{2}}^{\sigma_{3}} H(s, s) g^{+}(s) d s>1,
$$

and $\delta(t)$ is defined in (3.6), $A$ is defined in (3.2).

Therefore, for any $x \in K \cap \partial \Omega_{r}$, noticing (3.7), we get

$$
\begin{aligned}
\|T u\|= & \max _{t \in J}\left\{\mu \int_{0}^{1} \int_{0}^{1} H(t, s) H^{*}(s, \tau) a(s) b(\tau) u(s) u(\tau) d \tau d s\right. \\
& \left.+\lambda \int_{0}^{1} G(t, s) g(s) f(u(s)) d s\right\} \\
\geq & \max _{t \in J}\left\{\mu \int_{0}^{\sigma_{2}} H(t, s) a^{+}(s) u(s) \int_{0}^{1} H^{*}(s, \tau) b(\tau) u(\tau) d \tau d s\right. \\
& \left.+\lambda \int_{0}^{\sigma_{3}} G(t, s) g^{+}(s) f(u(s)) d s\right\} \\
\geq & \mu \min _{\frac{\sigma_{2}}{2} \leq t \leq \sigma_{2}} \int_{\frac{\sigma_{2}}{2}}^{\sigma_{2}} H(t, s) a^{+}(s) u(s) \int_{\frac{\sigma_{1}}{2}}^{\sigma_{1}} H^{*}(s, \tau) b^{+}(\tau) u(\tau) d \tau d s \\
& +\min _{\frac{\sigma_{2}}{2} \leq t \leq \sigma_{2}} \lambda \int_{\frac{\sigma_{3}}{2}}^{\sigma_{3}} H(t, s) g^{+}(s) f(u(s)) d s \\
\geq & \lambda \frac{\sigma_{2}}{2} \int_{\frac{\sigma_{3}}{2}}^{\sigma_{3}} H(s, s) g^{+}(s) \varepsilon_{3} u(s) d s \\
\geq & \lambda \frac{\sigma_{2}}{2} \varepsilon_{3} \min _{\frac{\sigma_{3}}{2} \leq t \leq \sigma_{3}} \delta(t) \int_{\frac{\sigma_{3}}{2}}^{\sigma_{3}} H(s, s) g^{+}(s) d s\|u\| \\
\geq & u \| .
\end{aligned}
$$




\section{Consequently,}

$$
\|T u\|>\|u\|, \quad u \in K \cap \partial \Omega_{r} .
$$

On the other hand, noticing that $0<\theta<1$, then it follows from $\left(H_{7}\right)$ that

$$
\lim _{u \rightarrow \infty} \frac{f(u)}{u} \leq \lim _{x \rightarrow \infty} \frac{k_{2} u^{\theta}}{u}=0
$$

which shows that there exists a $R^{\prime}>r$ such that

$$
f(u) \leq \varepsilon_{4} u, \quad u \geq R^{\prime}
$$

where $\varepsilon_{4}$ satisfies

$$
3 \lambda \varepsilon_{4} \int_{D(u)} H(s, s) g^{+}(s) d s<1
$$

and

$$
D(u)=\left\{t \in[0, \xi]: u(t)>R^{\prime}\right\}
$$

Let

$$
M=\lambda \max _{\|u\|_{P C}=R^{\prime}} f(u) \int_{0}^{\xi} H(s, s) g^{+}(s) d s
$$

Then it is not difficult to see that $M<+\infty$.

Choosing $\max \left\{R^{\prime}, 3 M\right\}<R<(3 A)^{-1}$, then $M<\frac{R}{3}$.

Now, choosing $u \in K \cap \partial \Omega_{R}$ arbitrarily, and letting $\bar{u}(t)=\min \left\{u(t), R^{\prime}\right\}$, then $\bar{u}(t) \leq R^{\prime}$. Therefore, for $t \in D(u)$, we get $R^{\prime}<u(t) \leq\|u\|_{P C}=R, \forall t \in D(u)$. By the choice of $R^{\prime}$, for $t \in D(u)$, we have $f(u) \leq \varepsilon_{4} R$.

Therefore, for any $u \in K \cap \partial \Omega_{R}$, we get

$$
\begin{aligned}
(T u)(t)= & \mu \int_{0}^{1} \int_{0}^{1} H(t, s) H^{*}(s, \tau) a(s) b(\tau) u(s) u(\tau) d \tau d s+\lambda \int_{0}^{1} H(t, s) g(s) f(u(s)) d s \\
= & \mu \int_{0}^{\xi} \int_{0}^{1} H(t, s) H^{*}(s, \tau) a^{+}(s) b(\tau) u(s) u(\tau) d \tau d s \\
& -\mu \int_{\xi}^{1} \int_{0}^{1} H(t, s) H^{*}(s, \tau) a^{-}(s) b(\tau) u(s) u(\tau) d \tau d s \\
& +\lambda \int_{0}^{\xi} H(t, s) g^{+}(s) f(x(s)) d s-\lambda \int_{\xi}^{1} G(t, s) g^{-}(s) f(u(s)) d s \\
\leq & \mu \int_{0}^{\xi} \int_{0}^{1} H(t, s) H^{*}(s, \tau) a^{+}(s) b(\tau) u(s) u(\tau) d \tau d s \\
& +\lambda \int_{0}^{\xi} H(t, s) g^{+}(s) f(u(s)) d s \\
= & \mu \int_{0}^{\xi} \int_{0}^{\xi} H(t, s) H^{*}(s, \tau) a^{+}(s) b^{+}(\tau) u(s) u(\tau) d \tau d s
\end{aligned}
$$




$$
\begin{aligned}
& -\mu \int_{0}^{\xi} \int_{0}^{\xi} H(t, s) H^{*}(s, \tau) a^{+}(s) b^{-}(\tau) u(s) u(\tau) d \tau d s \\
& +\lambda \int_{0}^{\xi} H(t, s) g^{+}(s) f(u(s)) d s \\
& \leq \mu \int_{0}^{\xi} \int_{0}^{\xi} H(s, s) H^{*}(s, \tau) a^{+}(s) b^{+}(\tau) u(s) u(\tau) d \tau d s \\
& +\lambda \int_{0}^{\xi} H(s, s) g^{+}(s) f(u(s)) d s \\
& \leq \mu \int_{0}^{\xi} \int_{0}^{\xi} H(s, s) H^{*}(s, \tau) a^{+}(s) b^{+}(\tau) u(s) u(\tau) d \tau d s \\
& +\lambda \int_{D(x)} H(s, s) g^{+}(s) f(u(s)) d s+\lambda \int_{[0, \xi] \backslash D(u)} H(s, s) g^{+}(s) f(u(s)) d s \\
& \leq \mu \int_{0}^{\xi} \int_{0}^{\xi} H(s, s) H^{*}(s, \tau) a^{+}(s) b^{+}(\tau) u(s) u(\tau) d \tau d s \\
& +\lambda \int_{D(x)} H(s, s) g^{+}(s) f(u(s)) d s+\lambda \int_{0}^{\xi} H(s, s) g^{+}(s) f(\bar{u}(s)) d s \\
& \leq \mu \int_{0}^{\xi} \int_{0}^{\xi} H(s, s) H^{*}(s, \tau) a^{+}(s) b^{+}(\tau) d \tau d s\|u\|^{2} \\
& +\lambda \varepsilon_{4} \int_{D(u)} H(s, s) g^{+}(s) d s\|u\|+M \\
& <\frac{1}{3}\|u\|+\frac{1}{3}\|u\|+\frac{1}{3}\|u\| \\
& =\|u\| \text {, }
\end{aligned}
$$

which shows that

$$
\|T u\|<\|u\|, \quad \forall u \in K \cap \partial \Omega_{R} .
$$

Let

$$
\lambda^{* *}=\frac{\eta}{2 M_{\eta} \int_{0}^{\xi} H(s, s) g^{+}(s) d s}, \quad \mu^{* *}=\frac{1}{2 A^{*} R}<\frac{1}{2 A^{*} \eta},
$$

where $0<\eta<r$, and $A^{*}$ is defined in (3.3).

We define $M_{\eta}$ by

$$
M_{\eta}=\max _{0 \leq u \leq \eta}\{f(u)\}>0 .
$$

Noticing $\left(H_{2}\right)$ and $\left(H_{3}\right)$, then $M_{\eta}$ is defined well and $M_{\eta}>0$. So, for any $u \in K \cap \partial \Omega_{\eta}$, similarly to the proof of (3.4), we get

$$
\begin{aligned}
(T u)(t) \leq & \mu \int_{0}^{\xi} \int_{0}^{\xi} H(s, s) H^{*}(s, \tau) a^{+}(s) b^{+}(\tau) u(s) u(\tau) d \tau d s \\
& +\lambda \int_{0}^{\xi} H(s, s) g^{+}(s) f(u(s)) d s
\end{aligned}
$$




$$
\begin{aligned}
& \leq \mu\|u\|^{2} A^{*}+\lambda \int_{0}^{\xi} H(s, s) g^{+}(s) d s M_{\eta} \\
& \leq \mu^{* *}\|u\|^{2} A^{*}+\lambda^{* *} \int_{0}^{\xi} H(s, s) g^{+}(s) d s M_{\eta} \\
& \leq \frac{1}{2}\|u\|+\frac{1}{2}\|u\| \\
& =\|u\|,
\end{aligned}
$$

which shows

$$
\|T u\| \leq\|u\|, \quad u \in K \cap \partial \Omega_{\eta} .
$$

Therefore, applying Lemma 2.4 to (3.13), (3.15) and (3.16) shows that $T$ has at least two fixed points in $K \cap\left(\bar{\Omega}_{R} \backslash \bar{\Omega}_{r}\right)$ and $K \cap\left(\Omega_{r} \backslash \Omega_{\eta}\right)$. Thus it follows that system (1.9) admits at least two positive solutions.

Theorem 3.2 Assume that $\left(H_{1}\right)-\left(H_{8}\right)$ hold. If $0<\theta<1$, there exist $\mu_{0}>0$ and $\lambda_{0}>0$ such that system (1.9) has three positive solutions for $\mu \in\left(0, \mu_{0}\right]$ and $\lambda \in\left(0, \lambda_{0}\right]$, with $k_{2}$ satisfying

$$
0<k_{2}<\frac{\left(2 \mu A^{*}\right)^{\theta-1}}{2 \lambda \int_{0}^{\xi} H(s, s) \omega^{+}(s) d s} .
$$

Proof Firstly, since $0<\theta<1$, by $\left(H_{7}\right)$, we get

$$
\lim _{u \rightarrow 0} \frac{f(u)}{u} \geq \lim _{u \rightarrow 0} \frac{k_{1} u^{\theta}}{u}=+\infty .
$$

Then there exists sufficiently small $r>0$ with $0<r<\left(2 \lambda k_{2} \int_{0}^{\xi} H(s, s) \omega^{+}(s) d s\right)^{\frac{1}{1-\theta}}$, such that

$$
f(u) \geq \varepsilon_{3} u, \quad 0 \leq u \leq r,
$$

where $\varepsilon_{3}$ satisfies (3.12). And hence, for $u \in \partial \Omega_{r}$, similar to the proof of (3.12), we obtain

$$
\|T u\|>\|u\|, \quad \forall u \in \partial \Omega_{r} .
$$

Secondly, there exists a sufficiently large $R_{1}>\max \left\{\left(2 \mu A^{*}\right)^{-1},\left(\mu B^{*}\right)^{-1}\right\}$ such that

$$
\mu B^{*} R_{1}>1 .
$$

Then, for $u \in \partial \Omega_{R_{1}}$, similar to the proof of (3.11), we get

$$
\|T u\| \geq\|u\|, \quad \forall u \in \partial \Omega_{R_{1}} .
$$

Thirdly, for any $\mu>0, \lambda>0$, we choose $R$ satisfying

$$
\left(2 \lambda k_{2} \int_{0}^{\xi} H(s, s) \omega^{+}(s) d s\right)^{\frac{1}{1-\theta}}<R<\left(2 \mu A^{*}\right)^{-1} .
$$


Then, for $u \in \partial \Omega_{R}$, it follows from (2.8) and $\left(H_{7}\right)$ that

$$
\begin{aligned}
& (T u)(t)=\mu \int_{0}^{1} \int_{0}^{1} H(t, s) H^{*}(s, \tau) a(s) b(\tau) u(s) u(\tau) d \tau d s \\
& +\lambda \int_{0}^{1} H(t, s) \omega(s) f(u(s)) d s \\
& =\mu \int_{0}^{\xi} \int_{0}^{1} H(t, s) H^{*}(s, \tau) a^{+}(s) b(\tau) u(s) u(\tau) d \tau d s \\
& -\mu \int_{\xi}^{1} \int_{0}^{1} H(t, s) H^{*}(s, \tau) a^{-}(s) b(\tau) u(s) u(\tau) d \tau d s \\
& +\lambda \int_{0}^{\xi} H(t, s) \omega^{+}(s) f(u(s)) d s-\lambda \int_{\xi}^{1} H(t, s) \omega^{-}(s) f(u(s)) d s \\
& \leq \mu \int_{0}^{\xi} \int_{0}^{1} H(t, s) H^{*}(s, \tau) a^{+}(s) b(\tau) u(s) u(\tau) d \tau d s \\
& +\lambda \int_{0}^{\xi} H(t, s) \omega^{+}(s) f(u(s)) d s \\
& =\mu \int_{0}^{\xi} \int_{0}^{\xi} H(t, s) H^{*}(s, \tau) a^{+}(s) b^{+}(\tau) u(s) u(\tau) d \tau d s \\
& -\mu \int_{0}^{\xi} \int_{0}^{\xi} H(t, s) H^{*}(s, \tau) a^{+}(s) b^{-}(\tau) u(s) u(\tau) d \tau d s \\
& +\lambda \int_{0}^{\xi} H(t, s) \omega^{+}(s) f(u(s)) d s \\
& \leq \mu \int_{0}^{\xi} \int_{0}^{\xi} H(s, s) H^{*}(s, \tau) a^{+}(s) b^{+}(\tau) u(s) u(\tau) d \tau d s \\
& +\lambda \int_{0}^{\xi} H(s, s) \omega^{+}(s) f(u(s)) d s \\
& \leq \mu \int_{0}^{\xi} \int_{0}^{\xi} H(s, s) H^{*}(s, \tau) a^{+}(s) b^{+}(\tau) u(s) u(\tau) d \tau d s \\
& +\lambda \int_{0}^{\xi} H(s, s) \omega^{+}(s) k_{2} u(s) d s \\
& \leq \mu \int_{0}^{\xi} \int_{0}^{\xi} H(s, s) H^{*}(s, \tau) a^{+}(s) b^{+}(\tau) d \tau d s\|u\|^{2} \\
& +\lambda \int_{0}^{\xi} H(s, s) \omega^{+}(s) k_{2} d s\|u\|^{\theta} \\
& =\mu A^{*}\|u\|^{2}+\lambda \int_{0}^{\xi} H(s, s) \omega^{+}(s) k_{2} d s\|u\|^{\theta} \\
& <\frac{1}{2}\|u\|+\frac{1}{2}\|u\| \\
& =\|u\| \text {. }
\end{aligned}
$$

\section{Consequently,}

$\|T u\|<\|u\|, \quad \forall u \in \partial \Omega_{R}$. 
Finally, choose a number $r_{1} \in\left(0, \frac{r}{2}\right)$. Noticing that $f(u)>0$ for all $u>0$, we can define, for $r_{1}>0$,

$$
\alpha_{r_{1}}=\max _{0 \leq u \leq r_{1}}\{f(u)\}
$$

It follows (3.23) that $f(u) \leq \alpha_{r_{1}}$ for $0 \leq u \leq r_{1}$. Let

$$
\mu_{0}=\frac{1}{2 A^{*} r_{1}}, \quad \lambda_{0}=\frac{r_{1}}{2 \int_{0}^{\xi} H(s, s) \omega^{+}(s) d s \alpha_{r_{1}}} .
$$

Then, for $u \in \partial \Omega_{r_{1}}$ and $0<\mu \leq \mu_{0}, 0<\lambda \leq \lambda_{0}$, similar to the proof of (3.15), we obtain

$$
\|T u\| \leq\|u\|, \quad \forall u \in \partial \Omega_{r_{1}} .
$$

Summing up we can show that $T$ has three fixed point $u_{1} \in \bar{\Omega}_{R_{1}} \backslash \bar{\Omega}_{R}, u_{2} \in \Omega_{R} \backslash \bar{\Omega}_{r}$ and $u_{3} \in \Omega_{r} \backslash \Omega_{r_{1}}$ by applying Lemma 2.5 to (3.18), (3.20), (3.22) and (3.25). And hence, if $0<\theta<1$, there exist $\mu_{0}>0$ and $\lambda_{0}>0$ such that system (1.9) has at least three positive solutions for $0<\mu \leq \mu_{0}$ and $0<\lambda \leq \lambda_{0}$, with $k_{2}$ satisfying (3.17). The gives the proof of Theorem 3.2.

\section{Remarks and comments}

In this section, we offer some remarks and comments of the associated system (1.9).

Remark 4.1 In Theorem 3.1 and Theorem 3.2, we generalize the results of [38-43] in three main directions:

(i) $g, h \in L^{1}[0,1]$ are nonnegative on $J$, not only $g \equiv 0$ and $h \equiv 0$ on $J$.

(ii) Multi-parameter case is in order.

(iii) The method used in the present paper is complete different from those in [33-38].

Remark 4.2 Comparing with Wang and An [44], the main features of this paper are as follows.

(i) The solvable intervals of positive parameter $\lambda_{i}(i=1,2,3)$ are given.

(ii) Nonlocal conditions are considered.

(iii) Triple positive solutions are obtained.

Remark 4.3 It is not difficult to see that the details of the proof of (3.14) are very different from that of (3.4). And the idea of the proof of (3.14) comes from Theorem 3.2 of [54].

Remark 4.4 Some ideas of dealing with parameters in Theorem 3.1 come from Theorems 2.1-2.4 in [55], but there are very few papers in the literature considering the multiplicity of positive solutions for second-order impulsive differential systems, especially in the case the weight functions $a(t), b(t)$ and $g(t)$ change sign on $[0,1]$.

Remark 4.5 It is not difficult to see that the conditions $\left(H_{2}\right)$ and $\left(H_{7}\right)$ play an important role in the proof of Theorem 3.1, and there are many functions satisfying $\left(H_{2}\right)$ and $\left(H_{7}\right)$, for example

$$
f(u)=u^{\delta}, \quad 0<\delta<+\infty .
$$


Remark 4.6 If we, respectively, replace the condition $\left(H_{2}\right)$ by

$\left(H_{2}^{\prime}\right) f \in C\left(\mathcal{R}^{+}, \mathcal{R}^{+}\right)$,

then we cannot guarantee $\sigma_{\eta}>0, M>0$ and $\alpha_{r_{1}}>0$, which are, respectively, defined in Theorem 3.1 and Theorem 3.2. Further, we cannot obtain the results of Theorem 3.1 and Theorem 3.2.

Remark 4.7 Similarly, one can consider the multiplicity of positive solutions for the following second-order nonlocal differential systems:

$$
\left\{\begin{array}{l}
-u^{\prime \prime}=a(t) \varphi u+\lambda \omega(t) f(u), \quad 0<t<1, \\
-\varphi^{\prime \prime}=\mu b(t) u, \quad 0<t<1, \\
u^{\prime}(0)=0, \quad u(1)=\int_{0}^{1} g(s) u(s) d s \\
\varphi(0)=\varphi(1)=\int_{0}^{1} h(s) \varphi(s) d s
\end{array}\right.
$$

where $\lambda>0$ and $\mu>0$ are two parameters, $a(t), \omega(t), b(t)$ change sign on $[0,1]$.

On the other hand, we conjecture that the conclusions in Theorem 3.1 and Theorem 3.2 also hold for the following systems:

$$
\left\{\begin{array}{l}
-u^{\prime \prime}=a(t) \varphi u+\lambda \omega(t) f(u), \quad 0<t<1, \\
-\varphi^{\prime \prime}=\mu b(t) u, \quad 0<t<1, \\
u(0)=u(1)=\int_{0}^{1} g(s) u(s) d s \\
\varphi(0)=\varphi(1)=\int_{0}^{1} h(s) \varphi(s) d s,
\end{array}\right.
$$

and

$$
\left\{\begin{array}{l}
-u^{\prime \prime}=a(t) \varphi u+\lambda \omega(t) f(u), \quad 0<t<1, \\
-\left(g(t) \varphi^{\prime}(t)\right)^{\prime}=\mu b(t) u, \quad 0<t<1, \\
u(0)=u(1)=\int_{0}^{1} g(s) u(s) d s, \\
a \varphi(0)-b \lim _{t \rightarrow 0^{+}} g(t) \varphi^{\prime}(t)=\int_{0}^{1} h(s) \varphi(s) d s, \\
a \varphi(1)+b \lim _{t \rightarrow 1^{-}} g(t) \varphi^{\prime}(t)=\int_{0}^{1} h(s) \varphi(s) d s,
\end{array}\right.
$$

where $\lambda>0$ and $\mu>0$ are two parameters, $a, b>0, g \in C^{1}([0,1],(0,+\infty)), a(t), \omega(t), b(t)$ change sign on $[0,1]$.

\section{An example}

To illustrate how our main results can be used in practice, we present an example.

Example 5.1 Consider the following system:

$$
\left\{\begin{array}{l}
-u^{\prime \prime}=a(t) \varphi u+\lambda \omega(t) u^{\theta}, \quad 0<t<1, \\
-\varphi^{\prime \prime}=\mu b(t) u, \quad 0<t<1, \\
u(0)=\int_{0}^{1} s^{3} u(s) d s, \quad u^{\prime}(1)=0 \\
\varphi(0)=\varphi(1)=\int_{0}^{1} s \varphi(s) d s
\end{array}\right.
$$


where $0<\theta<1$ and

$$
\begin{aligned}
& a(t)= \begin{cases}\frac{2048}{3}\left(\frac{1}{3}-t\right), & t \in\left[0, \frac{1}{3}\right], \\
-\frac{1}{16}\left(t-\frac{1}{3}\right), & t \in\left[\frac{1}{3}, 1\right],\end{cases} \\
& \omega(t)= \begin{cases}\frac{64}{\sqrt{3}}\left(\frac{1}{3}-t\right), & t \in\left[0, \frac{1}{3}\right], \\
-\frac{1}{16}\left(t-\frac{1}{3}\right), & t \in\left[\frac{1}{3}, 1\right],\end{cases} \\
& b(t)= \begin{cases}\frac{128}{3}\left(\frac{1}{3}-t\right), & t \in\left[0, \frac{1}{3}\right], \\
-\frac{1}{16}\left(t-\frac{1}{3}\right), & t \in\left[\frac{1}{3}, 1\right],\end{cases} \\
& g(t)=t^{3}, \quad h(t)=t .
\end{aligned}
$$

Conclusion. System (5.1) admits at least two positive solution for $0<\lambda \leq 0.091,0<\mu \leq$ 0.025 .

For convenience, we give a corollary of Proposition 2.3 in [40].

Corollary 5.1 Consider the following system:

$$
\left\{\begin{array}{l}
-x^{\prime \prime}=k(t) x^{\alpha}, \quad 0<t<1, \\
x(0)=\int_{0}^{1} g(t) x(t) d t, \quad x^{\prime}(1)=0
\end{array}\right.
$$

where $\alpha>0$ with $\alpha \neq 1, k(t)$ satisfying the changing sign condition,

$$
\begin{cases}k(t) \geq 0, & t \in[0, \xi] \\ k(t) \leq 0, & t \in[\xi, 1]\end{cases}
$$

and

$$
c_{1} x^{\alpha} \leq f(x)=x^{\alpha} \leq c_{2} x^{\alpha}, \quad c_{1}, c_{2}>0 .
$$

If there exists $0<\sigma<\xi$ such that

$$
c_{1} \frac{\xi-\sigma}{1-\xi} \sigma^{\alpha+1} \mu^{2} k^{+}\left(\xi-\frac{\xi-\sigma}{1-\xi} \tau\right) \geq c_{2} \xi^{\alpha} k^{-}(\xi+\tau), \quad \tau \in[0,1-\xi]
$$

then the following inequalities hold:

$$
\begin{aligned}
\sigma^{\alpha} \mu^{2} \int_{\sigma}^{\xi} H(t, s) k^{+}(s) d s & \geq \frac{c_{2}}{c_{1}} \xi^{\alpha} \int_{\xi}^{1} H(t, s) k^{-}(s) d s, \\
\sigma^{\alpha} \mu^{2} \int_{\sigma}^{\xi} G(t, s) k^{+}(s) d s & \geq \frac{c_{2}}{c_{1}} \xi^{\alpha} \int_{\xi}^{1} G(t, s) k^{-}(s) d s .
\end{aligned}
$$

Proof Similar to the proof of Proposition 2.3 in [40], one can prove

$$
G(t, \eta-\mu \xi) \geq \sigma G(t, \eta+\xi), \quad \xi \in[0,1-\eta] .
$$


And hence, it follows from (2.4) that

$$
\begin{aligned}
H(t, \eta-\mu \xi) & =G(t, \eta-\mu \xi)+\frac{1}{1-\varrho} \int_{0}^{1} G(\eta-\mu \xi, \tau) x(\tau) d \tau \\
& =G(t, \eta-\mu \xi)+\frac{1}{1-\varrho} \int_{0}^{1} G(\tau, \eta-\mu \xi) x(\tau) d \tau \\
& \geq \sigma G(t, \eta+\xi)+\frac{\sigma}{1-\varrho} \int_{0}^{1} G(\tau, \eta+\xi) x(\tau) d \tau \\
& =\sigma\left[G(t, \eta+\xi)+\frac{1}{1-\varrho} \int_{0}^{1} G(\tau, \eta+\xi) x(\tau) d \tau\right] \\
& =\sigma H(t, \eta+\xi), \quad \xi \in[0,1-\eta] .
\end{aligned}
$$

Next, letting $s=\eta-\frac{\xi-\sigma}{1-\xi} \xi, \xi \in[0,1-\eta]$, we get

$$
\int_{\sigma}^{\eta} H(t, s) K^{+}(s) d s=\frac{\xi-\sigma}{1-\xi} \int_{0}^{1-\eta} H\left(t, \eta-\frac{\xi-\sigma}{1-\xi} \xi\right) K^{+}\left(\eta-\frac{\xi-\sigma}{1-\xi} \xi\right) d \xi
$$

letting $s=\eta+\xi, \xi \in[0,1-\eta]$, we have

$$
\int_{\eta}^{1} H(t, s) K^{-}(s) d s=\int_{0}^{1-\eta} H(t, \eta+\xi) K^{-}(\eta+\xi) d \xi
$$

Finally, by integrating in $\xi$ both sides of (5.4) from 0 to $1-\eta$, it follows that the inequality (5.5) holds.

Similarly, one can show that the inequality (5.6) holds.

Remark 5.1 It is not difficult to see that Proposition 2.3 of [45] plays key roles in the proofs of the main results of [44] and [45]. However, it is invalid for nonlocal problems; for details, see the proof of Corollary 5.1 .

Proof of Example 5.1 From the definitions of $a(t), b(t)$ and $g(t)$, we know that $\xi=\frac{1}{3}$.

Step 1. We show that $\left(H_{4}\right)$ holds. For fixed $c_{1}=c_{2}=1, \sigma_{1}=\frac{1}{4}, \mu=1$ and $\alpha=1$, (5.4) is equivalent to the following inequality:

$$
\frac{3}{128} b^{+}\left(\frac{1}{3}-\frac{1}{8} \tau\right) \geq b^{-}\left(\frac{1}{3}+\tau\right), \quad \tau \in\left[0, \frac{2}{3}\right] \text {. }
$$

Letting $\frac{1}{3}-\frac{1}{8} \tau=\zeta$, it follows from (5.7) that

$$
\frac{3}{128} b^{+}(\zeta) \geq b^{-}\left(\frac{8}{3}-8 \zeta\right), \quad \zeta \in\left[\frac{1}{4}, \frac{1}{3}\right]
$$

According to the definition of $b(t)$, the inequality (5.8) holds clearly. And hence it follows from (5.6) that $\left(H_{4}\right)$ is reasonable.

Step 2. We prove the condition $\left(H_{5}\right)$ holds. Similar to the proof of Step 1 , letting $c_{1}=1$, $c_{2}=\frac{16}{\rho}, \sigma_{2}=\frac{1}{4}, \mu=1$ and $\alpha=1$, by (5.5) we get

$$
\frac{1}{4} \int_{\frac{1}{4}}^{\frac{1}{3}} H(t, s) a^{+}(s) d s \geq \frac{16}{3 \rho} \int_{\frac{1}{3}}^{1} H(t, s) a^{-}(s) d s
$$


Further, from the above inequality, it follows that

$$
\begin{aligned}
& \frac{1}{4} \int_{\frac{1}{4}}^{\frac{1}{3}} H(t, s) \frac{3 \rho}{16} a^{+}(s) d s \geq \int_{\frac{1}{3}}^{1} H(t, s) a^{-}(s) d s \\
& \quad \Leftrightarrow \quad \frac{1}{4} \int_{\frac{1}{4}}^{\frac{1}{3}} H(t, s)\left(\min _{s \in\left[\frac{1}{4}, \frac{1}{3}\right]} H^{*}(s, s)\right) a^{+}(s) d s \geq \int_{\frac{1}{3}}^{1} H(t, s) a^{-}(s) d s \\
& \Rightarrow \quad \frac{1}{4} \int_{\frac{1}{4}}^{\frac{1}{3}} H(t, s) H^{*}(s, s) a^{+}(s) d s \geq \int_{\frac{1}{3}}^{1} G(t, s) a^{-}(s) d s \\
& \quad \Rightarrow \quad \frac{1}{4} \int_{\frac{1}{4}}^{\frac{1}{3}} H(t, s) H^{*}(s, s) a^{+}(s) d s>\frac{1}{3} \int_{\frac{1}{3}}^{1} H(t, s) a^{-}(s) d s
\end{aligned}
$$

which shows that $\left(H_{5}\right)$ holds.

Step 3. Similar to the proof of Step 1, choosing $c_{1}=1, c_{2}=2, \sigma_{3}=\frac{1}{4}, \mu=1$ and $\alpha=\frac{1}{2}$, then $\left(H_{8}\right)$ holds.

Step 4. It is easy to see by direct calculating that

$$
\begin{aligned}
& \varrho=\int_{0}^{1} g(s) d s=\int_{0}^{1} s^{3} d s \frac{1}{4}, \quad v=\int_{0}^{1} h(s) d s=\int_{0}^{1} s d s=\frac{1}{2} \\
& \int_{0}^{\xi} g^{+}(s) d s=\int_{0}^{\frac{1}{3}} \frac{64}{\sqrt{3}}\left(\frac{1}{3}-s\right) d s=\frac{32}{9 \sqrt{3}} \\
& A^{*}=\int_{0}^{\xi} \int_{0}^{\xi} H(s, s) H^{*}(s, \tau) a^{+}(s) b^{+}(\tau) d \tau d s \approx 79.91 .
\end{aligned}
$$

Let $\eta=\frac{1}{4}$. Then we get

$$
M_{\eta}=\max _{0 \leq u \leq \frac{1}{4}}\{\sqrt{u}\}=\frac{1}{2}
$$

Therefore, we get

$$
\lambda^{* *}=\frac{\eta}{2 M_{\eta} \int_{0}^{\xi} H(s, s) g^{+}(s) d s} \approx 0.091, \quad \mu^{* *}=\frac{1}{3 A^{*} R} \approx 0.025
$$

Hence, by (II) of Theorem 3.1 the conclusion follows, and the proof is complete.

\section{Conclusion}

In this paper, we obtained several sufficient conditions for the multiplicity of positive solutions for a class of second-order nonlocal indefinite differential systems. Our results will be a useful contribution to the existing literature on the topic of second-order nonlocal indefinite differential systems. 


\section{Funding}

This work is sponsored by the National Natural Science Foundation of China (11401031), the Beijing Natural Science Foundation (1163007), the Scientific Research Project of Construction for Scientific and Technological Innovation Service Capacity (KM201611232017) and the teaching reform project of Beijing Information Science \& Technology University (2018JGYB41).

Availability of data and materials

Not applicable.

\section{Ethics approval and consent to participate}

Not applicable.

\section{Competing interests}

The authors declare that there is no conflict of interest regarding the publication of this manuscript. The authors declare that they have no competing interests.

\section{Consent for publication}

Not applicable.

\section{Authors' contributions}

All authors contributed equally and read and approved the final version of the manuscript.

\section{Publisher's Note}

Springer Nature remains neutral with regard to jurisdictional claims in published maps and institutional affiliations.

\section{Received: 17 January 2018 Accepted: 16 May 2018 Published online: 22 May 2018}

\section{References}

1. Chegis, R.Yu: Numerical solution of a heat conduction problem with an integral boundary condition. Liet. Mat. Rink. 24, 209-215 (1984)

2. Ionkin, N.I: Solution of a boundary value problem in heat conduction theory with nonlocal boundary conditions. Differ. Equ. 13, 294-304 (1977)

3. Sun, Y:: Optimal existence criteria for symmetric positive solutions to a three-point boundary value problem. Nonlinear Anal. 66, 1051-1063 (2007)

4. Hao, X., Zuo, M., Liu, L.: Multiple positive solutions for a system of impulsive integral boundary value problems with sign-changing nonlinearities. Appl. Math. Lett. 82, 24-31 (2018)

5. Mao, J., Zhao, Z.: The existence and uniqueness of positive solutions for integral boundary value problems. Bull. Malays. Math. Sci. Soc. 34, 153-164 (2011)

6. Hao, X., Liu, L., Wu, Y.: Positive solutions for second order impulsive differential equations with integral boundary conditions. Commun. Nonlinear Sci. Numer. Simul. 16, 101-111 (2011)

7. Hao, X., Liu, L.: Multiple monotone positive solutions for higher order differential equations with integral boundary conditions. Bound. Value Probl. 2014, 74 (2014)

8. Ahmad, B., Alsaedi, A., Alghamdi, B.S.: Analytic approximation of solutions of the forced Duffing equation with integral boundary conditions. Nonlinear Anal., Real World Appl. 9, 1727-1740 (2008)

9. Hao, X., Liu, L., Wu, Y.: Iterative solution to singular $n$ th-order nonlocal boundary value problems. Bound. Value Probl. $2015,125(2015)$

10. Feng, M., Du, B., Ge, W.: Impulsive boundary value problems with integral boundary conditions and one-dimensional p-Laplacian. Nonlinear Anal. 70, 3119-3126 (2009)

11. Hao, X., Liu, L., Wu, Y., Xu, N.: Multiple positive solutions for singular $n$ th-order nonlocal boundary value problems in Banach spaces. Comput. Math. Appl. 61, 1880-1890 (2011)

12. Jiang, J., Liu, L., Wu, Y.: Second-order nonlinear singular Sturm-Liouville problems with integral boundary conditions. Appl. Math. Comput. 215, 1573-1582 (2009)

13. Zhang, X., Ge, W.: Symmetric positive solutions of boundary value problems with integral boundary conditions. Appl. Math. Comput. 219, 3553-3564 (2012)

14. Zhang, X.: Exact interval of parameter and two infinite families of positive solutions for a nth order impulsive singular equation. J. Comput. Appl. Math. 330, 896-908 (2018)

15. Liu, L., Hao, X., Wu, Y.: Positive solutions for singular second order differential equations with integral boundary conditions. Math. Comput. Model. 57, 836-847 (2013)

16. Hao, X., Liu, L., Wu, Y., Sun, Q.: Positive solutions for nonlinear nth-order singular eigenvalue problem with nonlocal conditions. Nonlinear Anal. 73, 1653-1662 (2010)

17. Sun, F., Liu, L., Zhang, X., Wu, Y.: Spectral analysis for a singular differential system with integral boundary conditions Mediterr. J. Math. 13, 4763-4782 (2016)

18. Zhang, X., Feng, M., Ge, W.: Existence result of second-order differential equations with integral boundary conditions at resonance. J. Math. Anal. Appl. 353, 311-319 (2009)

19. Boucherif, A.: Second-order boundary value problems with integral boundary conditions. Nonlinear Anal. 70, 364-371 (2009)

20. Feng, M., Ji, D., Ge, W.: Positive solutions for a class of boundary-value problem with integral boundary conditions in Banach spaces. J. Comput. Appl. Math. 222, 351-363 (2008)

21. Feng, M., Zhang, X.: Time-map analysis to establish the exact number of positive solutions of one-dimensional prescribed mean curvature equations. Bound. Value Probl. 2014, 193 (2014) 
22. Liu, L., Sun, F., Zhang, X., Wu, Y.: Bifurcation analysis for a singular differential system with two parameters via to degree theory. Nonlinear Anal., Model. Control 22, 31-50 (2017)

23. Hao, X., Wang, H., Liu, L., Cui, Y.: Positive solutions for a system of nonlinear fractional nonlocal boundary value problems with parameters and p-Laplacian operator. Bound. Value Probl. 2017, 182 (2017)

24. Zhang, X., Feng, M.: Transformation techniques and fixed point theories to establish the positive solutions of second order impulsive differential equations. J. Comput. Appl. Math. 271, 117-129 (2014)

25. Hao, X., Xu, N., Liu, L.: Existence and uniqueness of positive solutions for fourth-order $m$-point boundary value problems with two parameters. Rocky Mt. J. Math. 43,1161-1180 (2013)

26. Hao, X., Liu, L., Wu, Y.: On positive solutions of an $m$-point nonhomogeneous singular boundary value problem. Nonlinear Anal. 73, 2532-2540 (2010)

27. Zhang, X., Feng, M.: Double bifurcation diagrams and four positive solutions of nonlinear boundary value problems via time maps. Commun. Pure Appl. Anal. 17, 2149-2171 (2018)

28. Hung, K.-C., Wang, S.-H., Yu, C.-H.: Existence of a double S-shaped bifurcation curve with six positive solutions for a combustion problem. J. Math. Anal. Appl. 392, 40-54 (2012)

29. Sun, F., Liu, L., Wu, Y.: Infinitely many sign-changing solutions for a class of biharmonic equation with $p$-Laplacian and Neumann boundary condition. Appl. Math. Lett. 73, 128-135 (2017)

30. Liu, J., Zhao, Z: Multiple solutions for impulsive problems with non-autonomous perturbations. Appl. Math. Lett. 64 143-149 (2017)

31. Kong, L.: Second order singular boundary value problems with integral boundary conditions. Nonlinear Anal. 72 , 2628-2638 (2010)

32. Zhang, X., Feng, M.: Existence of a positive solution for one-dimensional singular $p$-Laplacian problems and its parameter dependence. J. Math. Anal. Appl. 413, 566-582 (2014)

33. Autuori, G., Cluni, F., Gusella, V., Pucci, P.: Mathematical models for nonlocal elastic composite materials. Adv. Nonlinear Anal. 6, 355-382 (2017)

34. Bisci, G.M., Radulescu, V., Servadei, R.: Variational Methods for Nonlocal Fractional Problems. Encyclopedia of Mathematics and Its Applications, vol. 162. Cambridge University Press, Cambridge (2016)

35. Bisci, G.M., Repovs, D.: On some variational algebraic problems. Adv. Nonlinear Anal. 2, 127-146 (2013)

36. Bisci, G.M., Repovs, D., Servadei, R.: Nontrivial solutions of superlinear nonlocal problems. Forum Math. 28, 1095-1110 (2016)

37. Padhi, S., Pati, S., Hota, D.K.: Positive solutions of boundary value problems with nonlinear nonlocal boundary conditions. Opusc. Math. 36, 69-79 (2016)

38. Ma, R., Han, X.: Existence and multiplicity of positive solutions of a nonlinear eigenvalue problem with indefinite weight function. Appl. Math. Comput. 215, 1077-1083 (2009)

39. López-Gómez, J., Tellini, A.: Generating an arbitrarily large number of isolas in a superlinear indefinite problem. Nonlinear Anal. 108, 223-248 (2014)

40. Boscaggin, A., Zanolin, F.: Second-order ordinary differential equations with indefinite weight: the Neumann boundary value problem. Ann. Mat. Pura Appl. 194, 451-478 (2015)

41. Boscaggin, A., Zanolin, F.: Positive periodic solutions of second order nonlinear equations with indefinite weight: multiplicity results and complex dynamics. J. Differ. Equ. 252, 2922-2950 (2012)

42. Sovrano, E., Zanolin, F.: Indefinite weight nonlinear problems with Neumann boundary conditions. J. Math. Anal. Appl. 452, 126-147 (2017)

43. Bravo, J.L., Torres, P.J.: Periodic solutions of a singular equation with indefinite weight. Adv. Nonlinear Stud. 10, 927-938 (2010)

44. Wang, F., An, Y:: On positive solutions for a second order differential system with indefinite weight. Appl. Math. Comput. 259, 753-761 (2015)

45. Yao, Q.: Existence and multiplicity of positive radial solutions for a semilinear elliptic equation with change of sign. Appl. Anal. 80, 65-77 (2001)

46. López-Gómez, J., Omari, P., Rivetti, S.: Positive solutions of one-dimensional indefinite capillarity-type problems: a variational approach. J. Differ. Equ. 262, 2335-2392 (2017)

47. López-Gómez, J., Omari, P., Rivetti, S.: Bifurcation of positive solutions for a one-dimensional indefinite quasilinear Neumann problem. Nonlinear Anal. 155, 1-51 (2017)

48. Berestycki, H., Capuzzo-Dolcetta, I., Nirenberg, L.: Variational methods for indefinite superlinear homogeneous elliptic problems. Nonlinear Differ. Equ. Appl. 2, 553-572 (1995)

49. Berestycki, H., Capuzzo-Dolcetta, I., Nirenberg, L.: Superlinear indefinite elliptic problems and nonlinear Liouville theorems. Topol. Methods Nonlinear Anal. 4, 58-78 (2002)

50. Chen, W., Li, C.: Indefinite elliptic problems in a domain. Discrete Contin. Dyn. Syst., Ser. A 3, 333-340 (1997)

51. Amann, H., López-Gómez, J.: A priori bounds and multiple solutions for superlinear indefinite elliptic problems. J. Differ. Equ. 146, 336-374 (1998)

52. Feng, M.: Existence of symmetric positive solutions for a boundary value problem with integral boundary conditions. Appl. Math. Lett. 24, 1419-1427 (2011)

53. Guo, D., Lakshmikantham, V.: Nonlinear Problems in Abstract Cones. Academic Press, New York (1988)

54. Zhang, X., Feng, M., Ge, W.: Existence results for nonlinear boundary-value problems with integral boundary conditions in Banach spaces. Nonlinear Anal. 69, 3310-3321 (2008)

55. Zhang, X., Feng, M.: Multi-parameter, impulsive effects and positive periodic solutions of first-order functional differential equations. Bound. Value Probl. 2015, 137 (2015) 